\title{
Measurement of Microvibration by Using Dual-Cavity Fiber Fabry-Perot Interferometer for Structural Health Monitoring
}

\author{
Dae-Hyun Kim, ${ }^{1}$ Jin-Hyuk Lee, ${ }^{2}$ and Byung-Jun $\mathrm{Ahn}^{3}$ \\ ${ }^{1}$ Department of Mechanical and Automotive Engineering, Seoul National University of Science and Technology, 172 Gongneung 2-dong, \\ Nowon-gu, Seoul 139-743, Republic of Korea \\ ${ }^{2}$ Graduate School of Energy \& Environment, Seoul National University of Science and Technology, 172 Gongneung 2-dong, Nowon-gu, \\ Seoul 139-743, Republic of Korea \\ ${ }^{3}$ Graduate School of NID Fusion Technology, Seoul National University of Science and Technology, 172 Gongneung 2-dong, Nowon-gu, \\ Seoul 139-743, Republic of Korea
}

Correspondence should be addressed to Dae-Hyun Kim; dkim@seoultech.ac.kr

Received 4 December 2012; Accepted 14 March 2013; Published 3 March 2014

Academic Editor: Gyuhae Park

Copyright (C) 2014 Dae-Hyun Kim et al. This is an open access article distributed under the Creative Commons Attribution License, which permits unrestricted use, distribution, and reproduction in any medium, provided the original work is properly cited.

\begin{abstract}
Extensive researches have recently been performed to study structural integrity using structural vibration data measured by instructure sensors. A fiber optic sensor is one of candidates for the in-structure sensors because it is low in cost, light in weight, small in size, resistant to EM interference, long in service life, and so forth. Especially, an interferometric fiber optic sensor is very useful to measure vibrations with high resolution and accuracy. In this paper, a dual-cavity fiber Fabry-Perot interferometer was proposed with a phase-compensating algorithm for measuring micro-vibration. The interferometer has structurally two arbitrary cavities; therefore the initial phase difference between two sinusoidal signals induced from the interferometer was also arbitrary. In order to do signal processing including an arc-tangent method, a random value of the initial phase difference is automatically adjusted to the exact 90 degrees in the phase-compensating algorithm part. For the verification of the performance of the interferometer, a simple vibration-test was performed to measure micro-vibration caused by piezoelectric transducer (PZT). As an experimental result, the interferometer attached on the PZT successfully measured the $50 \mathrm{~Hz}$-vibration of which the absolute displacement oscillated between $-424 \mathrm{~nm}$ and $+424 \mathrm{~nm}$.
\end{abstract}

\section{Introduction}

Advanced sensor and structural monitoring technology can play an important role in prioritizing repair and rehabilitation process, improving the cost effectiveness of inspection and maintenance, and ultimately enhancing the longevity and safety of large-scale mechanical systems. Extensive researches have recently been performed to study structural integrity using structural vibration data measured by instructure sensors $[1,2]$. One of the major obstacles preventing sensor-based monitoring is however the unavailability of reliable, easy-to-install, and cost-effective sensors. In particular, civil engineering structures place unique demands on sensors. Besides accuracy, sensors and their cables are expected to be reliable, low in cost, light in weight, small in size, resistant to EM interference, and long in service life. They are required to withstand harsh environments, be moisture-, explosion-, and lightning-proof, and corrosion-resistant. Furthermore, civil structures are usually very large, demanding easy cabling of the sensors. It is very difficult, if not impossible, for the currently available electric-type sensors to satisfy these demanding requirements.

Emerging fiber optic sensor technologies have shown great potential to overcome the difficulties associated with the conventional sensors. They are immune to EM noise and electric shock and thus can be used in explosion-prone areas. Several kinds of fiber optic sensors have been developed over the last two decades to take advantage of these merits [3-6]. There have also been many field applications of fiber optic 


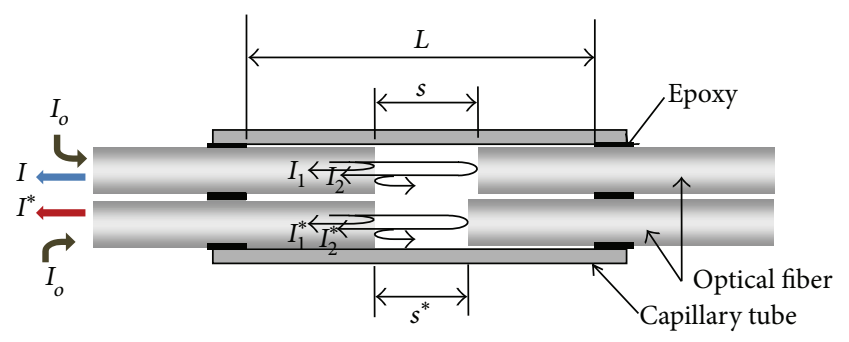

FIGURE 1: Schematic diagram of dual-cavity Fabry-Perot Interferometer.

sensors for health monitoring of engineering structures [79]. Among them, an interferometric fiber optic sensor is very useful to measure vibrations with high resolution and accuracy.

In this paper, a dual-cavity fiber Fabry-Perot interferometer was proposed with a phase-compensating algorithm. The sensor is structurally similar to a quadrature-phaseshifted fiber Fabry-Perot interferometer (QPS-FFPI); however, mechanical adjustment of two cavities is not needed because of the phase-compensating algorithm. Basically, the mechanical adjustment of two cavities is required in the QPSFFPI in order to take two sinusoidal signals which have an initial phase difference of exact 90 degrees $[10,11]$. However, the mechanical adjustment is very difficult, sometimes impossible. In the phase-compensating algorithm, a random value of the initial phase difference can be automatically adjusted to the exact 90 degrees. As a result, we can exactly measure the phase of the sinusoidal signal induced from the dual-cavity fiber Fabry-Perot interferometer by using an arctangent method. For the verification of the performance of the interferometer, a simple vibration test was performed to detect microvibration caused by piezoelectric transducer (PZT). As a result, it showed that the dual-cavity fiber FabryPerot interferometer successfully measured the microvibration with the assistance of the phase-compensating algorithm.

\section{Dual-Cavity Fiber Fabry-Perot Interferometer}

Theoretically, an interferometric fiber optic sensor generates a sinusoidal signal of which phase is changed periodically by the variation of external environment. A fiber FabryPerot interferometer is also one of interferometric fiber optic sensors and it can measure microvibration precisely because of its high sensitivity and accuracy. In this paper, two fiber Fabry-Perot interferometers are used as one sensor called a dual-cavity fiber Fabry-Perot interferometer. Figure 1 shows a sensor head of the dual-cavity fiber Fabry-Perot interferometer. As shown in Figure 1, two pairs of optical fibers in the sensor generate two sinusoidal signals because of optical interference. An initial phase difference between the output signals of $I$ and $I^{*}$ should be 90 degrees for using an arctangent method. So, the initial phase difference is adjusted to the exact 90 degrees in the case of QPS-FFPI by the

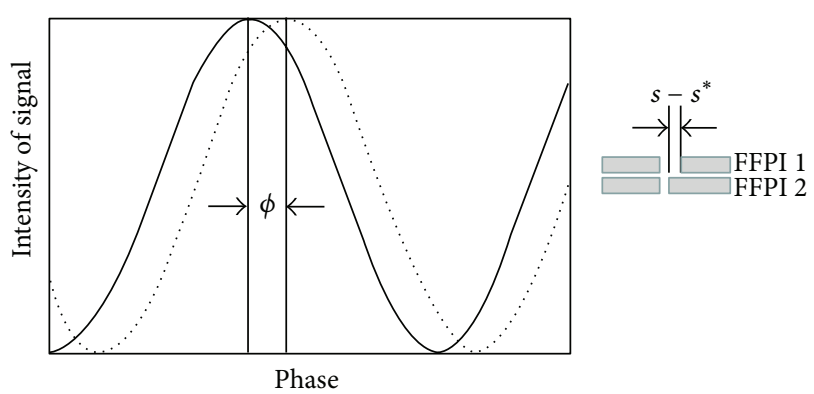

FFPI 1

FFPI 2

Figure 2: Characteristics of signals of dual-cavity Fabry-Perot Interferometer.

mechanical adjustment of the cavities of $s$ and $s^{*}$. However, the dual-cavity fiber Fabry-Perot interferometer has arbitrary values of $s$ and $s^{*}$; therefore, the output signals have an initial phase difference $(\phi)$ as a random value as shown in Figure 2. This condition is a major difference between the dual-cavity fiber Fabry-Perot interferometer and the conventional QPSFFPI.

\section{Signal Processing with Phase-Compensating Algorithm}

As mentioned in the previous section, the dual cavity fiber Fabry-Perot interferometer has an arbitrary value of the initial phase difference between two output sinusoidal signals. Therefore, it is needed for the measurement of displacement that the initial phase difference is forcibly adjusted to the exact 90 degrees. In this section, a signal processing part is introduced with a phase-compensating algorithm in which the initial phase difference is automatically changed to the exact 90 degrees. A detailed explanation is following. Firstly, the intensity signals of the two pairs of optical fibers with an arbitrary initial phase difference $(\phi)$ can be expressed in (1) and (2) as follows. Secondly, these two sinusoidal signals are needed to be normalized as shown in (3) and (4). Consider

$$
\begin{gathered}
I_{1}(t)=C_{1} \sin \left(2 \frac{2 \pi}{\lambda} s(t)\right)+C_{2}, \\
I_{2}(t)=C_{3} \sin \left(2 \frac{2 \pi}{\lambda} s(t)+\phi\right)+C_{4}, \\
\bar{I}_{1}(t)=\frac{I_{1}(t)-C_{2}}{C_{1}}=\sin \left(2 \frac{2 \pi}{\lambda} s(t)\right), \\
\bar{I}_{2}(t)=\frac{I_{2}(t)-C_{4}}{C_{3}}=\sin \left(2 \frac{2 \pi}{\lambda} s(t)+\phi\right),
\end{gathered}
$$

where $C_{1}$ and $C_{3}$ are amplitudes of sinusoidal signals and $C_{2}$ and $C_{4}$ are values of offsets. $s(t)$ is a cavity between two optical fibers of the interferometer as shown in Figure 1. Thirdly, the normalized signals $\left(\bar{I}_{1}, \bar{I}_{2}\right)$ and $\phi$ are used for generating a new sinusoidal signal of which wavelength is the same as the 


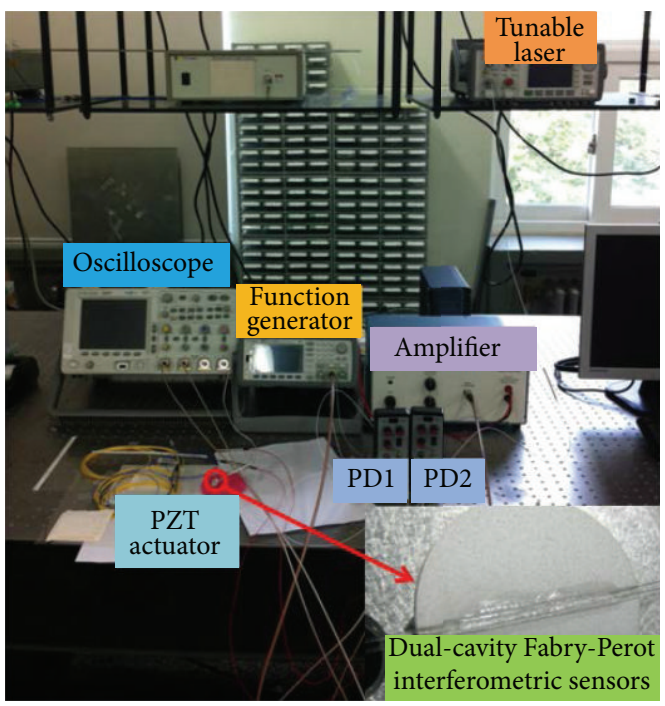

FIGURE 3: Experimental setup for measuring microvibration by using a dual-cavity fiber Fabry-Perot interferometer.

normalized signal of $\bar{I}_{1}$. The phase difference between the new sinusoidal signal $\left(\bar{I}_{3}\right)$ and $\bar{I}_{1}$ is exactly 90 degrees. The new sinusoidal signal $\left(\bar{I}_{3}\right)$ is expressed in

$$
\bar{I}_{3}=f\left(\bar{I}_{1}, \bar{I}_{2}, \phi\right)=\sin \left(2 \frac{2 \pi}{\lambda} s(t)+\frac{\pi}{2}\right)=\cos \left(2 \frac{2 \pi}{\lambda} s(t)\right) .
$$

The equations for calculating the displacement of $\Delta s(t)$ can be expressed as follows:

$$
\begin{gathered}
s(t)=\frac{\lambda}{4 \pi} \operatorname{unwrap}\left(\tan ^{-1}\left(\frac{\bar{I}_{1}}{\bar{I}_{3}}\right)\right) \\
\Delta s(t)=\frac{\lambda}{4 \pi} \Delta\left[\operatorname{unwrap}\left(\tan ^{-1}\left(\frac{\bar{I}_{1}}{\bar{I}_{3}}\right)\right)\right] .
\end{gathered}
$$

As a result, the displacement can be successfully measured by calculating the phase-shifting.

\section{Measurement of Microvibration}

4.1. Experimental Setup and Procedures. Figure 3 shows an experimental setup to measure microvibration by using the dual-cavity fiber Fabry-Perot interferometer with the phasecompensating algorithm. An actuating system is composed of a linear amplifier (PIEZO, EPA-104-230), a function waveform generator (AGILENT, 33522A), and PZT (FUJI CERAMICS, C82). A sensing system is also composed of a tunable laser (AGILENT, 81949A) as a laser source, two photodetectors (NEWFOCUS, 2117) as laser receivers, and a dual-cavity fiber Fabry-Perot interferometer (gauge length $=10.2 \mathrm{~mm}$ ) which is attached on the surface of the PZT as shown in Figure 3.

In detail, these optical components are combined by using one coupler and two circulators as shown in Figure 4. Two photodetectors are connected to an oscilloscope (AGILENT,

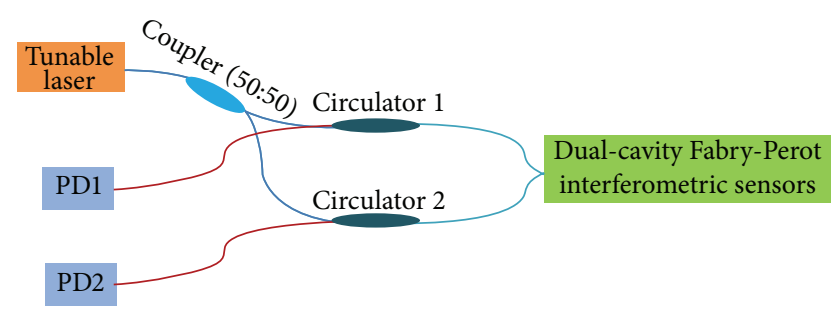

FIgURE 4: Experimental setup for driving optical devices of dualcavity fiber Fabry-Perot interferometer.

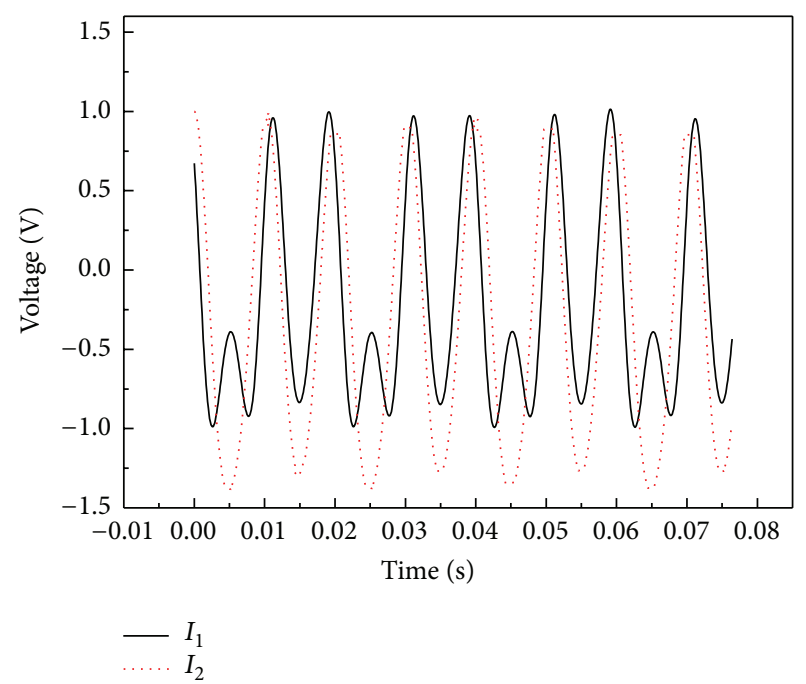

FIGURE 5: Two sinusoidal signals induced from a dual-cavity fiber Fabry-Perot interferometer.

DSO6034A) to observe and save the output signals induced from the dual-cavity fiber Fabry-Perot interferometer.

Experimental procedures are the following. Firstly, the wavelength of the tunable laser is set as $1542 \mathrm{~nm}$, and a $50 \mathrm{~Hz}-$ sinusoidal signal is generated from the function waveform generator. Secondly, the amplitude of the sinusoidal signal is forced to be gradually increased by controlling the gain value of the linear amplifier. Thirdly, two output signals induced from the dual-cavity fiber Fabry-Perot interferometer are measured by the oscilloscope. Finally, the absolute value and variation of the displacement are calculated in a signal processing software including the phase-compensating algorithm explained in the previous section.

4.2. Experimental Results. Figure 5 shows two sinusoidal signals induced from the dual-cavity fiber Fabry-Perot interferometer. A low pass filter of which the cut-off frequency was $190 \mathrm{~Hz}$ was applied to the signals for noise reduction. The first step of the signal processing was to normalize these two sinusoidal signals by using minimum and maximum values of the sinusoidal signals. The second step was to generate a new sinusoidal signal by using these two normalized sinusoidal signals and the initial phase difference $(\phi)$.

Figure 6 shows the normalized sinusoidal signals $\left(\bar{I}_{1}, \bar{I}_{2}\right)$ and the newly generated sinusoidal signal $\left(\bar{I}_{3}\right)$. It was also 


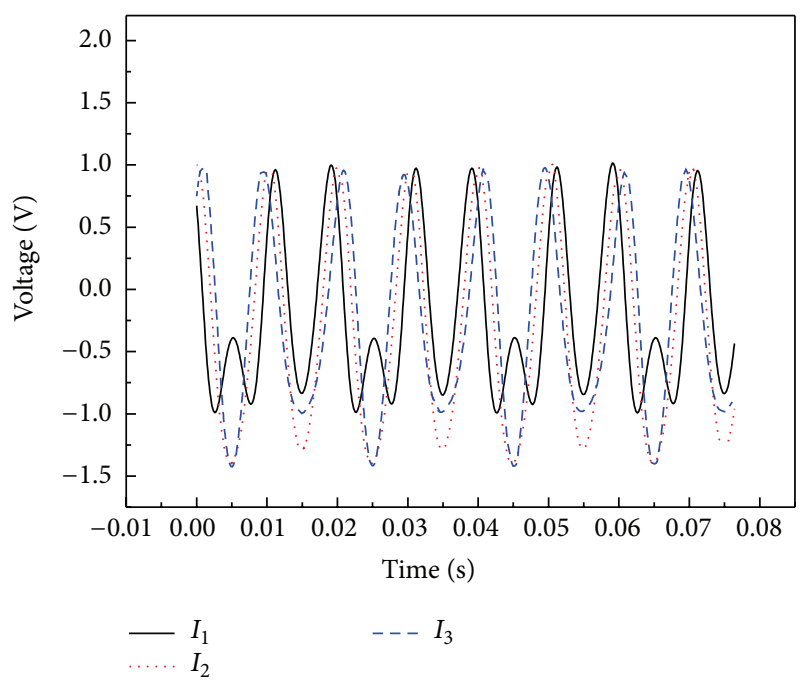

FIGURE 6: Two normalized sinusoidal signals and one newly generated signal for the signal processing.

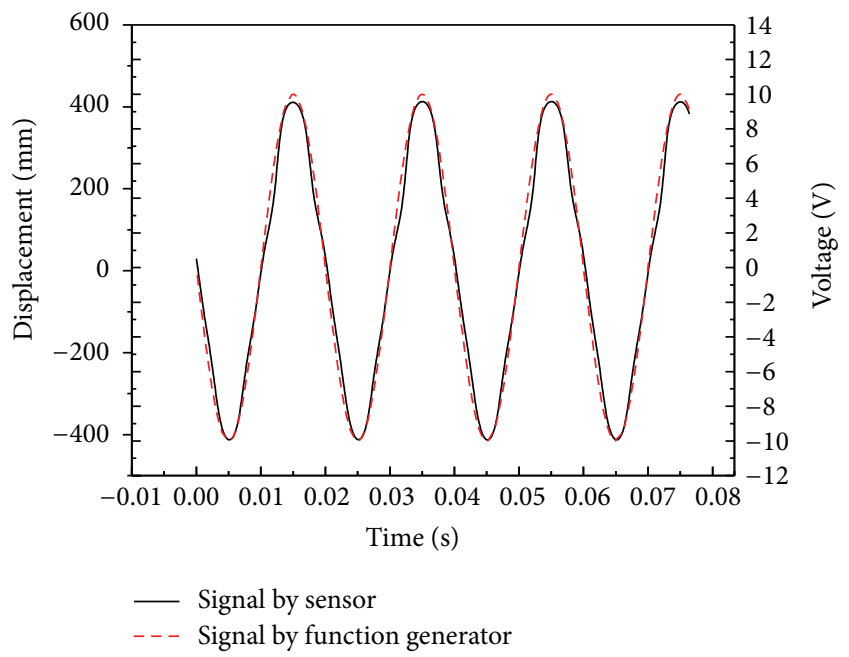

Figure 7: Absolute value of displacement induced by vibrationexcitation.

checked out that the phase difference between $\bar{I}_{1}$ and $\bar{I}_{3}$ was exactly 90 degrees. The phase of $\bar{I}_{1}$ or $\bar{I}_{2}$ and the absolute value of the displacement were calculated by using (6) as explained in Section 3. Figure 7 shows the calculated displacement which oscillates between $-424 \mathrm{~nm}$ and $+424 \mathrm{~nm}$. The frequency of the oscillating displacement was also checked by a spectrum analysis. Figure 8 shows the result of the spectrum analysis that the frequency is the same as the excitation frequency of $50 \mathrm{~Hz}$. From the experiment, it is verified that the dual-cavity fiber Fabry-Perot interferometer can successfully measure the microvibration by using the phase-compensating algorithm.

\section{Conclusions}

In this paper, a dual-cavity fiber Fabry-Perot interferometer was proposed with a phase-compensating algorithm. The

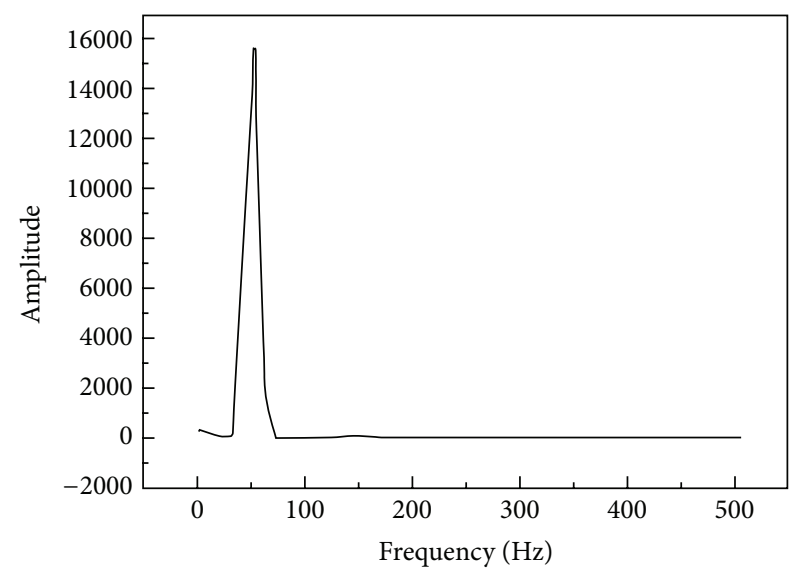

FIGURE 8: Spectrum analysis of the oscillating displacement.

dual-cavity fiber Fabry-Perot interferometer generates two sinusoidal signals of which initial phase difference was a random value. In the phase-compensating algorithm, the random value of the initial phase difference can be automatically adjusted to the exact 90 degrees. As a result, we can exactly measure the phase of the sinusoidal signal induced from the dual cavity fiber Fabry-Perot interferometer by using an arc-tangent method. For the verification of the performance of the interferometer, an experimental test was performed. In the experiment, a dual-cavity fiber Fabry-Perot interferometer was fabricated and attached on the surface of the PZT. The interferometer was excited by the PZT and two output sinusoidal signals were processed with the phasecompensating algorithm to measure the displacement of the PZT. As a result, the interferometer successfully measured the displacement induced by the PZT. In detail, the PZT led to $50 \mathrm{~Hz}$ mechanical vibration and the interferometer attached on the PZT successfully measured the same vibration of which the absolute displacement oscillated between $-424 \mathrm{~nm}$ and $+424 \mathrm{~nm}$. Furthermore, it is expected that the dualcavity fiber Fabry-Perot interferometer can be applied to more precise and accurate vibration measurement with the phase-compensating algorithm.

\section{Conflict of Interests}

The authors declare that there is no conflict of interests regarding the publication of this paper.

\section{Acknowledgments}

This research was supported by Basic Science Research Program through the National Research Foundation of Korea (NRF) funded by the Ministry of Education, Science and Technology (2010-0004312) and Basic Science Research Program through the National Research Foundation of Korea (NRF) funded by the Ministry of Education (2013R1A1A2007061). 


\section{References}

[1] M. Q. Feng, J.-M. Kim, and H. Xue, "Identification of a dynamic system using ambient vibration measurements," Journal of Applied Mechanics, vol. 65, no. 4, pp. 1010-1021, 1998.

[2] M. Q. Feng and D.-H. Kim, "Novel fiber optic accelerometer system using geometric moiré fringe," Sensors and Actuators A: Physical, vol. 128, no. 1, pp. 37-42, 2006.

[3] E. Udd, Fiber Optic Smart Structures, John Wiley \& Sons, New York, NY, USA, 1995.

[4] B. Lee, "Review of the present status of optical fiber sensors," Optical Fiber Technology, vol. 9, no. 2, pp. 57-79, 2003.

[5] D. H. Kim and M. Q. Feng, "Real-time structural health monitoring using a novel fiber optic accelerometer system," IEEE Sensors Journal, vol. 7, no. 4, pp. 536-543, 2007.

[6] J. Im, M. Kim, K. S. Choi, T. K. Hwang, and I. B. Kwon, "FBG sensor probes with silver epoxy for tracing the maximum strain of structures," Journal of KNST, vol. 33, no. 5, pp. 459-464, 2013.

[7] H.-N. Li, D.-S. Li, and G.-B. Song, "Recent applications of fiber optic sensors to health monitoring in civil engineering," Engineering Structures, vol. 26, no. 11, pp. 1647-1657, 2004.

[8] F. Ansari, "Fiber optic health monitoring of civil structures using long gage and acoustic sensors," Smart Materials and Structures, vol. 14, no. 3, pp. S1-S7, 2005.

[9] W. Moorman, L. Taerwe, W. De Waele, J. Degrieck, and J. Himpe, "Measuring ground anchor forces of a quay wall with Bragg sensors," Journal of Structural Engineering, vol. 131, no. 2, pp. 322-328, 2005.

[10] D. A. Jackson and J. D. C. Jones, "Interferometers optical fiber sensors," Optical Fiber Sensors: Systems and Applications, vol. 12, no. 4, pp. 277-281, 2001.

[11] K. A. Murphy, M. F. Gunther, A. M. Vengsarkar, and R. O. Claus, "Quadrature phase-shifted, extricsic fabry-perot optical fiber sensors," Optics Letters, vol. 16, no. 4, pp. 273-275, 1991. 

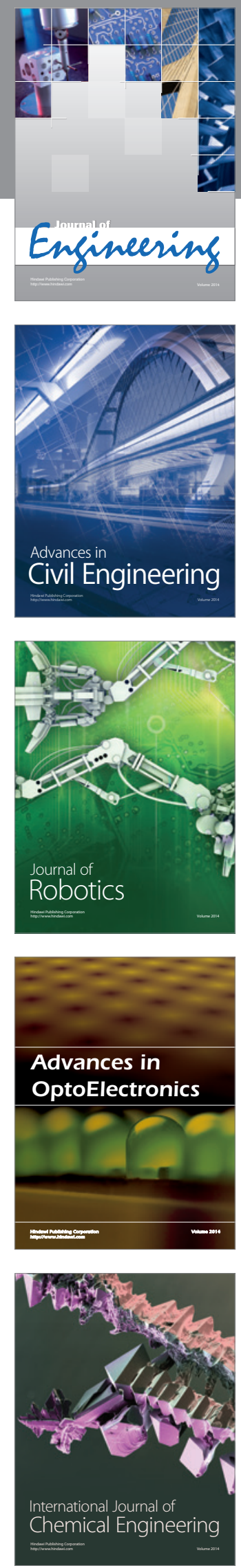

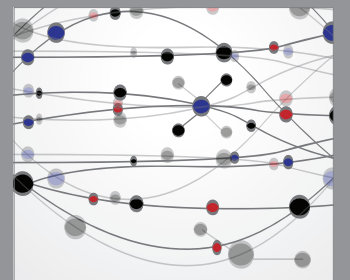

The Scientific World Journal
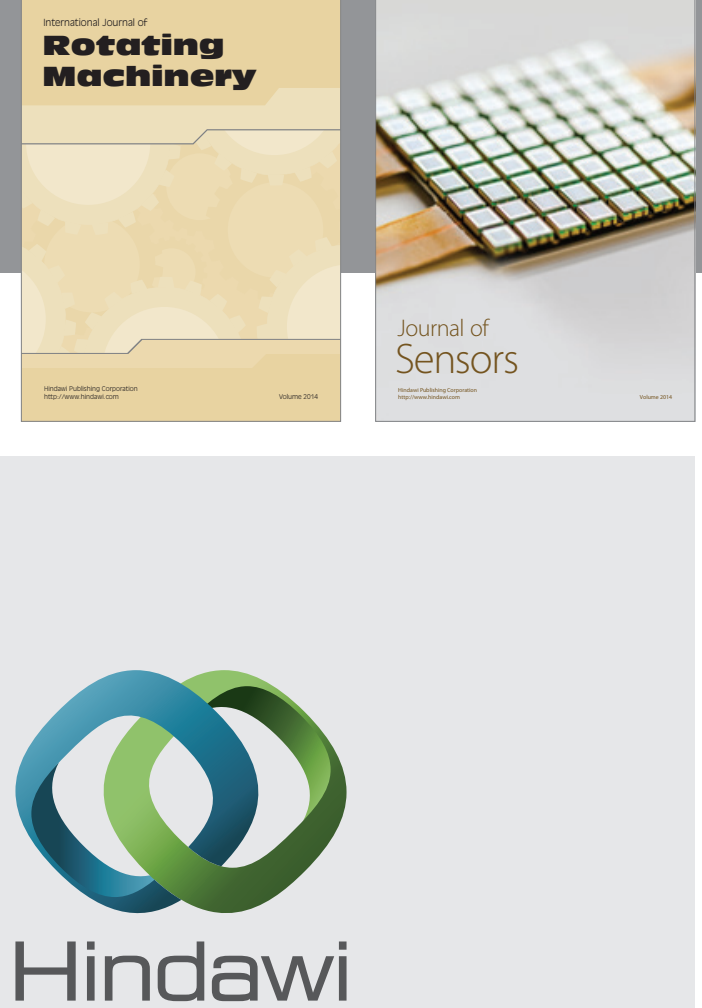

Submit your manuscripts at http://www.hindawi.com
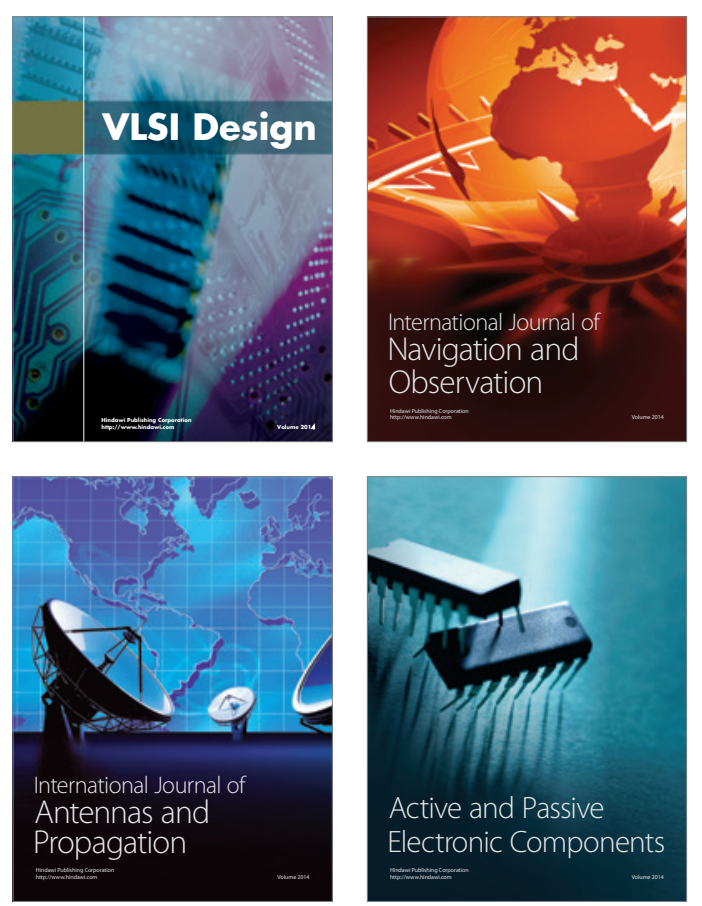
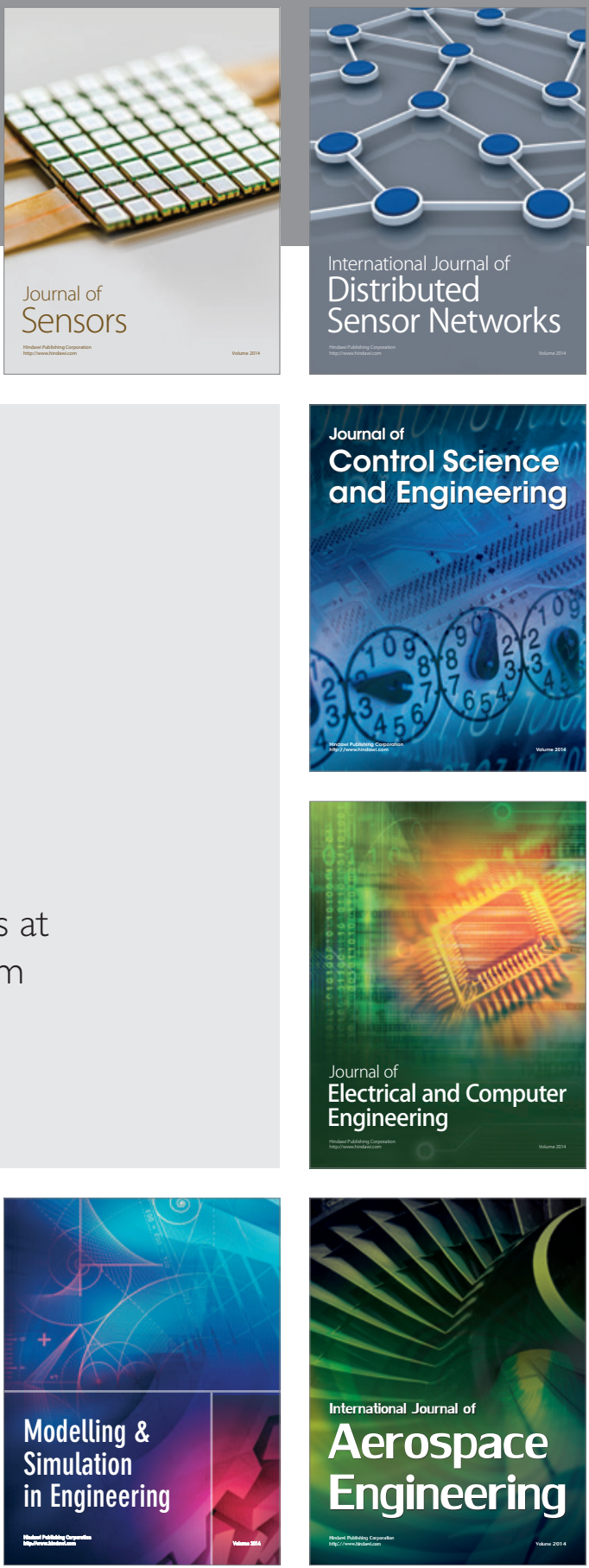

Journal of

Control Science

and Engineering
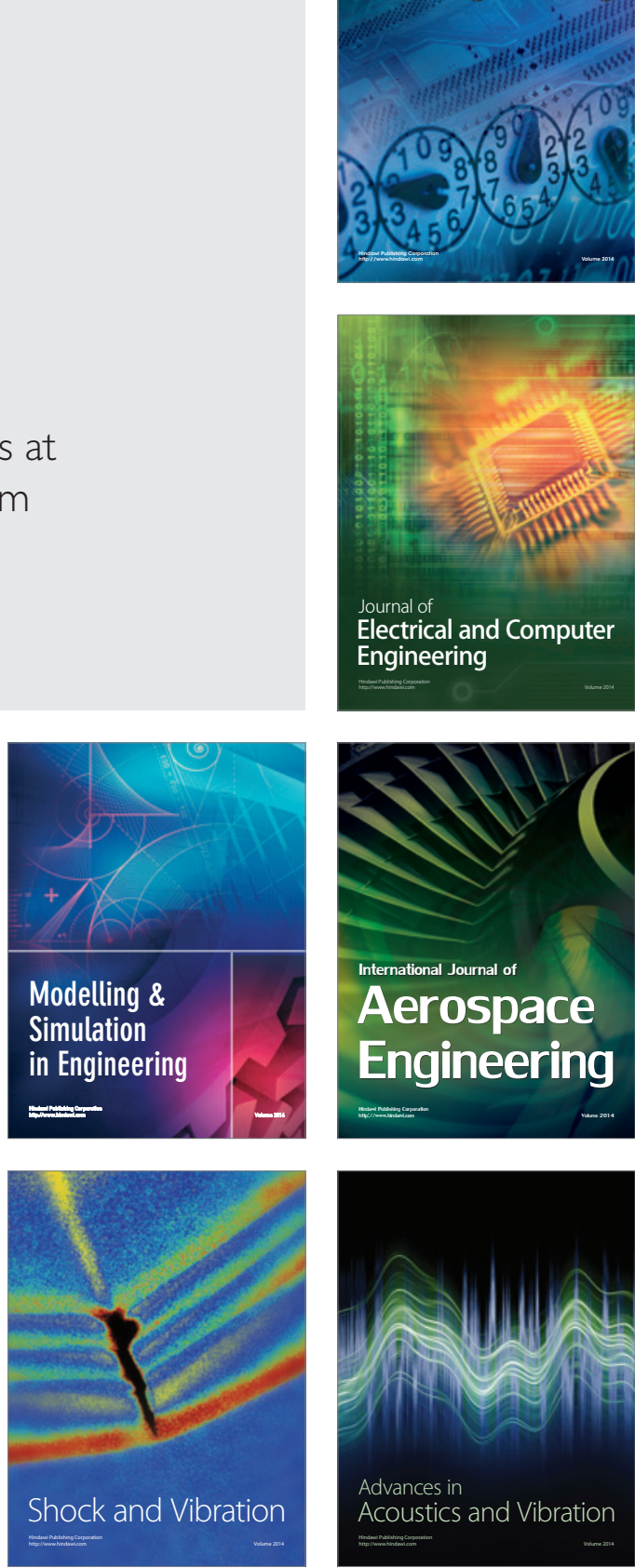